\title{
53. The Structure of Open Algebraic Surfaces and Its Application to Plane Curves
}

\author{
By Shuichiro TsunodA \\ Department of Mathematics, Osaka University \\ (Communicated by Kunihiko KodaIrA, M. J. A., April 13, 1981)
}

The purpose of this note is to outline our recent results on the structure of algebraic surfaces which may not be complete. Details will be published elsewhere.

1. A triple $(X, \bar{X}, D)$ is said to be a non-singular triple, if $\bar{X}$ is a complete non-singular surface over the field of complex numbers and if $D$ is a divisor with only simple normal crossings such that $X=\bar{X} \backslash D$. We denote by $K(\bar{X})$ the canonical divisor on $\bar{X}$. We define logarithmic $m$-genera $\bar{P}_{m}(X)$ and the logarithmic Kodaira dimension $\bar{\kappa}(X)$ by

(see [2]).

$$
\begin{aligned}
\bar{P}_{m}(X) & =\operatorname{dim} H^{0}(\bar{X}, m(K(\bar{X})+D)), \\
\bar{\kappa}(X) & =\kappa(K(\bar{X})+D, \bar{X})
\end{aligned}
$$

In general, let $\Delta$ be a divisor on $\bar{X}$ with $\kappa(\Delta, \bar{X}) \geq 0$. Then one has a $\boldsymbol{Q}$-divisor $\Delta^{+}$and an effective $\boldsymbol{Q}$-divisor $\Delta^{-}$such that

(1) $\Delta=\Delta^{+}+\Delta^{-}$,

(2) $\Delta^{+}$is semipositive (i.e. $\left(\Delta^{+}, \Gamma\right) \geq 0$ for all curves $\Gamma$ on $\bar{X}$ ),

(3) the intersection matrix of $\Delta^{-}$is negative-definite or $\Delta^{-}=0$,

(4) $\left(\Delta^{+}, \Delta^{-}\right)=0$.

This decomposition is unique and is called the Zariski decomposition of $\Delta$ (see [4] or [5]).

The main results are summarized as follows:

Theorem 1. If $\bar{\kappa}(X)=0$, then $\bar{P}_{i}(X)=1$ for some $i, 1 \leq i \leq 66$.

Theorem 2. If $\bar{\kappa}(X) \geq 0$ and if $D$ is connected, then $\overline{\bar{P}}_{12}(X)>0$.

We shall outline proofs of these theorems. A triple $(X, \bar{X}, D)$ is said to be almost minimal if the support of $(K(\bar{X})+D)^{-}$contains no exceptional curve of the 1st kind.

Lemma 3. Given a triple $(X, \bar{X}, D)$ with $\bar{\kappa}(X) \geq 0$, there exist an almost minimal triple $(Z, \bar{Z}, B)$ and a birational morphism $f: \bar{X} \rightarrow \bar{Z}$ having the following properties:

(1) $B=f_{*}(D)$,

(2) $(K(\bar{X})+D)^{+}=f^{*}\left((K(\bar{Z})+B)^{+}\right)$.

By the above lemma, it suffices to prove the theorems for almost minimal triples $(X, \bar{X}, D)$. We need the following

Proposition 4. If $(X, \bar{X}, D)$ is almost minimal, then $D-(K(\bar{X})+$ $\left.+D)^{-}\right)$is effective and $\left(\bar{X}, D-(K(\bar{X})+D)^{-}\right)$is a relatively minimal model 
of $(X, \bar{X}, D)$ in the sense of Kawamata [4].

Put $D_{\min }=D-(K(\bar{X})+D)^{-}$. By Kawamata [4], $n\left(K(\bar{X})+D_{\min }\right)$ is generated by global sections for $n \gg 0$.

Firstly, we shall prove Theorem 1. Mimicking an argument in Fujita [1], we can construct a non-singular triple $(\mathscr{X}, \bar{X}, \mathscr{D})$ and a morphism $\Pi: \bar{X} \rightarrow \bar{X}$ such that

(1) $\mathscr{D}=\Pi^{-1}(D)$,

(2) $\left.\Pi\right|_{\mathscr{X}}$ is an etale covering map,

(3) $\bar{P}_{1}(\mathfrak{X})=1$.

Furthermore, we can assume that a cyclic group $G$ acts on $\bar{X}$ in such a way that $\mathscr{D}$ is $G$-invariant and $\mathscr{X} / G$ is isomorphic to $X$. Let $\sigma$ be a generator of $G$. Then $\sigma$ gives rise to an automorphism of $H^{0}(\overline{\mathscr{X}}, K(\overline{\mathscr{X}})$ $+\mathscr{D})$ which will be denoted by $\sigma^{*}$. We denote the eigenvalue of $\sigma^{*}$ by $\alpha$. Then $\alpha$ is a primitive $n$-root of unity and we have $\bar{P}_{n}(X)=1$. On the other hand, by making use of the classification of the logarithmic $K 3$ surfaces (Iitaka [3]), we obtain $n \leq 66$.

Secondly, we shall prove Theorem 2.

(A) The case in which $\tilde{\kappa}(X)=0$. By a rather easy argument we can conclude that $\bar{P}_{12}(X)=1$.

(B) The case in which $\bar{\kappa}(X)=1$. By Kawamata [1], we have $\bar{P}_{12}(X)>0$.

(C) The case in which $\bar{\kappa}(X)=2$. We need the following

Proposition 5. If $(X, \bar{X}, D)$ is almost minimal, then $P_{n}(X)$ $=1 / 2\left(n K(\bar{X})-\left[-(n-1) D_{\min }\right]+\left[D_{\min }\right], \quad(n-1) K(\bar{X})-\left[-(n-1) D_{\min }\right]\right.$ $\left.+\left[D_{\min }\right]\right)+\chi\left(\mathcal{O}_{X}\right)+\varepsilon(n, D)$ for $n \geq 2$, where $\varepsilon(n, D)$ is a non-negative integer.

By the above proposition, we have $\bar{P}_{12}(X)>0$. Note that the proof of this part is somewhat complicated.

2. In this section, we shall outline a proof of the following

Theorem 6. Let $(x, y, z)$ be a system of homogeneous coordinates in $\boldsymbol{P}^{2}$. Let $C_{a, \alpha_{1}, \cdots, \alpha_{a}}\left(a \geq 3, \alpha_{1}, \cdots, \alpha_{a} \in C\right)$ be the curve defined by

$$
\begin{aligned}
\left(y^{a-1} z\right. & \left.-\prod_{i=1}^{a}\left(x-\alpha_{i} y\right)\right)^{a} z \\
& +\sum_{i=1}^{a-1}\left(\begin{array}{c}
a \\
i
\end{array}\right) x^{i} y^{a^{2}+1-a i-i}\left(y^{a-1} z-\prod_{i=1}^{a}\left(x-\alpha_{i} y\right)\right)^{i}+y^{a^{2+1}}=0 .
\end{aligned}
$$

The curve $C_{a, \alpha_{1}, \ldots, \alpha_{a}}$ has the following properties :

(1) $C \backslash\{0: 0: 1\} \cong A^{1}$,

(2) $\bar{\kappa}\left(\boldsymbol{P}^{2} \backslash C\right)=1$.

Conversely, the curve having the properties (1) and (2) is $C_{a, \alpha_{1}, \ldots, \alpha_{a}}$ up to projective equivalence for some $\left(\alpha, \alpha_{1}, \ldots, \alpha_{a}\right)$.

Let $\mu: \bar{X} \rightarrow \boldsymbol{P}^{2}$ be a composition of blow-ups such that $D=\mu^{-1}(C)$ has only simple normal crossings. We can assume that $\mu$ is shortest among such birational morphisms. We set $X=\boldsymbol{P}^{2} \backslash C$. We denote by $\Pi: \bar{X} \rightarrow \Delta$ 
a morphism associated with $|n(K(\bar{X})+D)|$ for $n \gg 0$. Since $X$ is affine, we have $\Pi(D)=\Delta$. This implies a general fiber of $\left.\Pi\right|_{X}$ is $\boldsymbol{G}_{m}^{1}$. Hence, there exist a Hirzebruch surface $\bar{Y}$ and a birational morphism $\rho: \bar{X} \rightarrow \bar{Y}$ such that $\Pi \cdot \rho^{-1}$ is a morphism. We put $\varphi=\Pi \cdot \rho^{-1}$ and denote by $f$ a general fiber of $\varphi$.

By taking a suitable $\bar{Y}$, we can assume that $\Gamma=\rho_{*}(D)$ is either

(i) a sum of a 2-section (i.e. an irreducible curve $\varepsilon$ with $(\varepsilon, f)=2$ ) and at most three fibers, or

(ii) a sum of two sections for the fibration $\varphi$ and at most three fibers.

Note that

(1) each irreducible component of $D$ has a nezative self-intersection number and

(2) the exceptional curve in $D$ is unique.

It follows from (1) and (2) that $\Gamma$ is a sum of two sections and three fibers.

Then, we can conclude that $(X, \bar{X}, D)$ is a resolution of $C_{a, \alpha_{1}, \ldots, \alpha a}$.

\section{References}

[1] T. Fujita: On Kaehler fiber spaces over curves. J. Math. Soc. Japan, 30, 779-794 (1978).

[2] S. Iitaka: On logarithmic Kodaira dimension of algebraic varieties. Complex Analysis and Algebraic Geometry. Iwanami, Tokyo, pp. 175-189 (1977).

[ 3 ] - : On logarithmic K3 surfaces. Osaka J. Math., 16, 675-705 (1979).

[4] Y. Kawamata: Classification of non-complete algebraic surfaces. Proc. Japan Acad., 54A, 133-135 (1978).

[5] O. Zariski: The theorem of Riemann Roch for high multiples of an effective divisor on an alegbraic surface. Ann. of Math., 76, 560-615 (1962). 